\title{
Kollektive als Zurechnungssubjekte
}

\author{
Joachim Renzikowski
}

\section{Vorbemerkung: Zum Begriff der Zurechnung}

Auf den ersten Blick scheint das Thema nicht viel mit der Normentheorie zu tun zu haben. Schließlich wird etwa die Frage, ob und inwieweit juristische Personen bestraft werden können, bislang nicht im Kontext der Unterscheidung von primären Verhaltens- und sekundären Sanktionsnormen diskutiert. Ein Bezug ergibt sich aus dem Begriff der "Zurechnung“. Denn alle Normen richten sich an Personen, d.h. Subjekte, deren „Handlungen einer Zurechnung fähig sind“. ${ }^{1}$ Der hierbei angesprochene Begriff der Zurechnung, wie er in einer bis auf Aristoteles zurückreichenden Tradition in der praktischen Philosophie der Aufklärung ausgearbeitet wurde, ${ }^{2}$ ist streng zu unterscheiden von der heute in der Strafrechtsdogmatik üblichen Verwendung dieses Ausdrucks. So bezeichnet die sog. „objektive Zurechnung" die Verwirklichung eines unerlaubten Risikos in einem rechtlich missbilligten Erfolg und beschreibt damit nichts anderes als einen unerwünschten Geschehensablauf, in den ein Mensch als potentieller Täter involviert ist, und der daher grundsätzlich Gegenstand von Verhaltensnormen sein kann. Aber die „objektive Zurechnung“ sagt nach ihrem Selbstverständnis nichts darüber aus, ob der Normadressat diesen Geschehensablauf auch hätte vermeiden können, denn das Schuldurteil kommt ja erst nach der Feststellung der Rechtswidrigkeit. Davon abgesehen knüpft sie an ein Verständnis von Kausalität als Äquivalenz aller Bedingungen an, welches etwa die Geburt eines Kindes zugleich als Verursachung seines Todes ansieht, so dass man sich fragen kann, ob es sich überhaupt um einen sinnvollen Begriff handelt. ${ }^{3}$

1 Kant, Metaphysik der Sitten (2. Aufl. 1798), in: Kants gesammelte Schriften. Hrsg. v. d. Königlich Preußischen Akademie der Wissenschaften, Band 6, 1907, S. 203 (223).

2 Allgemein dazu Aichele, in: Hilgendorf/Joerden (Hrsg.), Handbuch Rechtsphilosophie, 2017, S. $401 \mathrm{ff}$.

3 Zur Kritik s. etwa Aichele, ZStW 123 (2011), 260 ff. 
In der Einleitung zur Rechtslehre definiert Kant die Zurechnung als

„das Urteil, wodurch jemand als Urheber (causa libera) einer Handlung, die alsdann Tat (factum) heißt und unter Gesetzen steht, angesehen wird; welches, wenn es zugleich die rechtlichen Folgen aus dieser Tat bei sich führt, eine rechtskräftige (imputatio iudiciaria s. valida) ... sein würde. (...) Der rechtliche Effekt einer Verschuldung ist die Strafe (poena)."4

Tat ist

„eine Handlung, die unter den Gesetzen der Verbindlichkeit steht, folglich auf das Subjekt in derselben nach der Freiheit seiner Willkür betrachtet wird. Der Handelnde wird durch einen solchen Akt als Urheber der Wirkung betrachtet, und diese zusamt der Handlung selbst können ihm zugerechnet werden, wenn man vorher das Gesetz kennt, kraft welches auf ihnen eine Verbindlichkeit ruht." ${ }^{\text {"5 }}$

Eine Handlung ist demnach nur dann eine Tat, wenn sie unter eine Verhaltensnorm, sei es eine Norm des Rechts oder der Moral, subsumiert werden kann, ${ }^{6}$ womit wir wieder bei der Normentheorie sind: Wer kann Adressat einer Verhaltensnorm sein? Wem kann der Verstoß gegen eine Verhaltensnorm vorgeworfen werden? Eben diese Frage beantwortet die Zurechnung. Oder konkret, da es immer um Personen geht: Sind Personen nur Individuen, oder können auch Kollektive Personen sein?

Wie gesagt, charakterisiert die Zurechnungsfähigkeit die Person. Nach dem obigen Zitat fährt Kant fort: „Die moralische Persönlichkeit ist also nichts anderes als die Freiheit eines vernünftigen Wesens unter moralischen Gesetzen."7 Diese Freiheit taucht auch in dem Zurechnungsbegriff auf. Grundlage jeder Zurechnung und zugleich ihre Voraussetzung ist die von Kant so genannte „freie Willkür“. Unter Willkür versteht Kant die Fähigkeit, etwas „nach Belieben zu tun oder zu lassen“, verbunden „mit dem Bewußtsein des Vermögens seiner Handlung zur Hervorbringung des Objekts". ${ }^{8}$ Willkür setzt demnach neben der Vorstellung eines tatsächlich erreichbaren Handlungsziels die Kenntnis der zu seiner Erreichung geeigne-

4 Kant, MdS, AA VI, S. 227.

5 Kant, MdS, AA VI, S. 223.

6 Näher dazu Hruschka, in: Schröder (Hrsg.), Theorie der Interpretation vom Humanismus bis zur Romantik - Rechtswissenschaft, Philosophie, Theologie, 2001, S. 203 ff.; Aichele, JRE 15 (2007), $251 \mathrm{ff}$.

7 Kant, MdS, AA VI, S. 223.

8 Kant, MdS, AA VI, S. 213. 
ten Mittel, m.a.W. die prospektive Kenntnis der entsprechenden kausalen Prozesse voraus. Die Willkür ist „frei“, wenn sie in „Unabhängigkeit ihrer Bestimmung durch sinnliche Antriebe“ „zu Handlungen aus reinem Willen bestimmt werden“ kann. Hierin unterscheidet sich die „menschliche Willkür ..., welche durch Antriebe zwar affiziert, aber nicht bestimmt wird“, von der „tierische[n] Willkür (arbitrium brutum)“, die nur „durch Neigung (sinnlichen Antrieb, stimulus) bestimmbar ist".9 Dieser Zurechnungsbegriff schließt die Verantwortlichkeit eines Kollektivs nicht aus.

\section{Persona moralis simplex und Persona moralis composita}

Ein Subjekt, das in seinen Handlungen der Gesetzgebung der praktischen Vernunft folgen, d.h. zwischen verschiedenen denkbaren Handlungsmaximen auswählen und sich damit für oder gegen die Befolgung einer Norm entscheiden kann, ist eine Person. ${ }^{10}$ Der von Kant verwendete Ausdruck der "moralischen Persönlichkeit" ist eine direkte Übersetzung von Pufendorfs persona moralis.

Der Begriff der „moralischen Persönlichkeit“ verdeutlicht zunächst, dass man ,Person' nicht ohne weiteres mit ,Mensch` gleichsetzen kann. Nicht jeder Mensch ist schon per se zurechnungsfähig, man denke etwa an Kleinkinder oder Geisteskranke. Die Altersgrenzen für die Strafmündigkeit, oder die Gründe, die nach den $\mathbb{S}$ 20, 21 StGB die Zurechnungsfähigkeit aufheben oder einschränken, sind normative Setzungen. Eine Person ist somit kein Gegenstand der Empirie, sondern gehört in die Welt des Rechts bzw. der praktischen Philosophie. ${ }^{11}$ Auch muss man die Person von der physischen Substanz unterscheiden. Der physische Körper ,Mensch` ist es zwar, der ein bestimmtes Ereignis hervorbringt. Aber erst die freie Willkür, auf die die fragliche Handlung zurückgeht, ist hinreichende Bedingung eines Zurechnungsurteils. Aus dem Begriff der Zurechnung folgt aber nicht, dass diese freie Willkür nur an einen bestimmten individuellen physischen Körper gebunden sein kann. Die herkömmliche Redeweise von der ,natürlichen Person' im Gegensatz zur ,juristischen Person' ist deshalb ungenau. Klarer wäre die Unterscheidung zwischen einer persona moralis simplex zur Bezeichnung von Individuen und einer persona moralis

9 Ibid.

10 Näher dazu Aichele, in: Kaufmann/Renzikowski (Hrsg.), Zurechnung als Operationalisierung von Verantwortung, 2004, S. $247 \mathrm{ff}$.

11 So in aller Deutlichkeit Kelsen, Reine Rechtslehre, 1934, S. 52 f. 
composita zur Bezeichnung von Kollektiven. Die letztere Ausdrucksweise war in der Aufklärung nicht unüblich. Im Folgenden wird zu zeigen sein, wie diese persona moralis composita gebildet wird. ${ }^{12}$

Die rechtliche Verantwortlichkeit von Kollektiven und ihren Mitgliedern füreinander war allerdings kein neues Problem, sondern wurde seit dem Mittelalter diskutiert. ${ }^{13}$ Zu Beginn der Neuzeit befasste sich etwa Grotius im Zusammenhang mit seiner Lehre vom gerechten Krieg mit den Folgen staatlichen Unrechts. Die Schuld der obersten Staatsgewalt sollte nur dann auf die Untertanen übergehen, wenn sie in das Verbrechen eingewilligt oder auf Befehl des Herrschers selbst Unrecht getan hatten. ${ }^{14} \mathrm{Im}$ 17. und 18. Jahrhundert war die Vorstellung, dass auch Kollektivpersonen schuldhaft handeln können, in der Rechtsphilosophie und in der Rechtswissenschaft weit verbreitet. ${ }^{15}$

Soweit ersichtlich kann Pufendorf als Schöpfer der persona moralis composita gelten. Er schreibt:

„Eine zusammengesetzte moralische Person wird begründet, wenn mehrere menschliche Individuen sich so untereinander vereinigen, dass, was sie kraft derselben Vereinigung wollen und tun, für einen Willen und für eine Handlung und nicht für viele gehalten wird. Und dies wird dann als geschehen verstanden, wenn die Einzelnen ihren Willen dem Willen eines einzigen Menschen oder einer Versammlung so unterwerfen, dass sie für den Willen und die Handlung aller anerkennen und von anderen dafür gehalten haben wollen, was immer der- oder dieselbe bezüglich jenem, was sich auf die Natur dieser Vereinigung als solcher bezieht und mit ihrem Zweck übereinstimmt, entschieden und ausgeführt haben mag." 16

12 Dazu eingehend Aichele, JRE 16 (2008), 1 (3 ff.). Nebenbei zeigt sich hieran, dass die Entgegensetzung von naturalistischem und kommunikativ begründetem Strafrecht (so Dust, Täterschaft von Verbänden, 2019, S. 18 f.) - was immer das heißen soll - verfehlt ist.

13 Näher dazu Maihold, Strafe für fremde Schuld, 2005, S. 64 ff.

14 Grotius, De Jure Belli ac Pacis, Libri tres, editio nova, 1680, lib. II, cap. XXI, $\$ \mathbb{S} 7$, 17 f.; s. dazu auch Recknagel, Einheit des Denkens trotz konfessioneller Spaltung. Parallelen zwischen den Rechtslehren von Francisco Suárez und Hugo Grotius, 2010, S. $268 \mathrm{ff}$.

15 S. die Nachweise bei Aichele, JRE 16 (2008), 1 (7f.).

16 Pufendorf, De jure naturae et gentium, ed. secunda, 1684, lib. I, cap. 1, $\mathbb{1 3}$ : „Persona moralis composita constituitur, quando plura individua humana ita inter se uniuntur, ut quae vi istius unionis volunt aut agunt, pro una voluntate, unaque actione, non pro pluribus censeantur. Idque tunc fieri intelligitur, quando singuli voluntatem suam voluntati unius hominis aut consilii ita subjiciunt, ut pro omni- 
Wenn Pufendorf von einer "zusammengesetzten moralischen Person“ spricht, dann gibt es offensichtlich auch ,schlichte ${ }^{6}$ moralische Personen, die heutzutage so genannten ,natürlichen Personen' ${ }^{6}{ }^{17}$ Mehrere Individuen können nun durch Willensübereinkunft eine moralische Person begründen. Kraft dieser Übereinkunft wird der Wille jedes Einzelnen zum gemeinsamen Willen aller. Auf diese Weise bilden die Individuen in Beziehung auf die moralische Person ein einheitliches Ganzes, d.h., sie können im Hinblick auf ihre Handlungen im Rahmen dieser Gemeinschaft nicht unterschieden werden.

Dieses auf Leibnitz zurückgehende Argument der logischen Identität des Ununterscheidbaren findet sich ausdrücklich bei Nettelbladt, bei dem es heißt:

„Daher bilden viele menschliche Individuen eine moralische Person, oder aufgrund dieses zu prüfenden Prinzips eben nicht. So oft vieler einzelner Menschen Vorstellung, Willen und Kräfte auf dasselbe gerichtet werden, so sollen sie jedes Mal gleichermaßen als eine Person angesehen werden, und so sind sie die moralische Person. So werden nämlich ihre Vorstellung, ihre Willen und ihre Kräfte wie eine einzige Vorstellung, ein einziger Wille und eine einzige Kraft angesehen, und wenn sie sich auch von einem Mensch in der Zahl ihrer Körper unterscheiden, so ist dieser Unterschied nicht zu berücksichtigen. “18

Was also der persona moralis composita zugerechnet, d.h. auf ihren Willen zurückgeführt werden kann, kann aufgrund der formalen Identität in gleicher Weise allen ihren Teilen, also den einzelnen Individuen zugerechnet werden. Oder in der Terminologie Kants: Eine moralische Person ist dadurch gekennzeichnet, dass sie als "causa libera“ eine Handlung hervor-

um volunate $\&$ actione velint agnoscere, $\&$ ab aliis haberi, quicquid iste decreverit aut gesserit circa illa, quae ad unionis ejus naturam ut talem spectant, \& fini ejusdem congruunt." Übersetzung von Aichele, JRE 16 (2008), 1 (9).

17 S. dazu Auer, in: Gröschner/Kirste/Lembcke (Hrsg.), Person und Rechtsperson, 2015, S. $81 \mathrm{ff}$.

18 Nettelbladt, Systema elementare universae jurisprudentiae naturalis in usum praelectionum academicarum adornatum (5. Aufl. 1785), in: Ecole (Hrsg.), Christian Wolff, Gesammelte Werke, Abteilung III, Bd. 39, 1, 1997, 84 S. 48: „An plura individua humana moralem personam constituant, vel non, ex hoc principio diiudicandum. Quoties plurium individuorum humanorum intellectus, voluntates et vires tendunt ad idem, toties, quoad hoc idem, pro una persona sunt haben$\mathrm{da}$, sicque sunt persona moralis. Tum enim eorum intellectus, voluntates et vires sunt ut unus intellectus, una voluntas, et una vis, sicque ab uno homine non nisi in corporum numero differunt, quae differentia hic non est attentenda.“ 
bringen kann; sie hat also das Vermögen, zu handeln. Dieses Vermögen hat sie, weil sie einen gesetzgebenden Willen besitzt, d.h. aus einer Einheit verschiedener menschlicher Willen besteht. Ihre Taten können aber nicht nur ihr, d.h. dem Kollektiv zugerechnet werden, sondern sie können jedem Mitglied dieser moralischen Person zugerechnet werden, weil sie sich ihrer Art nach, d.h. nach der Beteiligung an der moralischen Person, nicht voneinander unterscheiden. Auch wenn die Teile einer moralischen Person einzelne physische Personen sind, stellt jede Handlung, die sie in ihrer Funktion als Teil der moralischen Person vornehmen, eine Handlung des Ganzen und somit auch jedes einzelnen Teiles dar.

„Insofern folglich eine physische Person Teil einer moralischen Person ist und dieser moralischen Person Taten zugerechnet werden, handelt es sich dabei nicht um fremde Taten, die dieser einzelnen physischen Person zugerechnet werden, auch wenn sie nicht deren physische Ursache war. “19

\section{Anwendungen}

\section{Mittäterschaft}

Ein erster Anwendungsbereich der persona moralis composita ist die Mittäterschaft. ${ }^{20}$ Die Deutung der Mittäterschaft als Personengemeinschaft, der das gemeinsame Werk als Ganzes zugerechnet wird, findet sich auch in der heutigen Beteiligungsformendogmatik, ${ }^{21}$ auch wenn die Vertreter dieser Ansicht nicht mit der persona moralis composita argumentieren. Der Haupteinwand lautet, es sei unklar, weshalb ein Einzelner als Mittäter für die eigenverantwortlichen Tatbeiträge der anderen mitverantwortlich sein solle.

19 Aichele, JRE 16 (2008), 1 (22).

20 So bereits Nettelbladt, Systema Elementare Iurisprudentiae Positivae Germanorum Communis Generalis, 1781, $₫ 877$; ebenso auch die Deutung von Haas, in: Kaufmann/Renzikowski (Hrsg.), Zurechnung und Verantwortung, ARSP-Beiheft 134, 2012, S. 125 (133).

21 S. etwa Joerden, Strukturen des strafrechtlichen Verantwortlichkeitsbegriffs: Relationen und ihre Verkettungen, 1988, S. 78 ff.; Renzikowski, Restriktiver Täterbegriff und fahrlässige Beteiligung, 1997, S. 100 f.; Heinrich, Rechtsgutszugriff und Entscheidungsträgerschaft, 2002, S. 287 f.; Mylonopoulos, GA 2011, 462 (464 f.); Stratenwerth/Kublen, Strafrecht, Allgemeiner Teil, 6. Aufl. 2011, $\mathbb{1} 12$ Rn. 77. 
Die Bildung einer Kollektivperson könne nur eine kollektive Verantwortlichkeit, nicht aber die Verantwortung eines Einzelnen begründen. ${ }^{22}$

Nun wird die persona moralis composita durch den übereinstimmenden Willen mehrerer Individuen gebildet. Diese Individuen können zwar als physische Körper voneinander und auch von dem Kollektiv unterschieden werden, aber darauf kommt es für die moralische Person nicht an. Es ist gerade der Witz der persona moralis composita, dass die Individuen in Beziehung auf die moralische Person nicht im Hinblick auf ihre Handlungen im Rahmen dieser Gemeinschaft voneinander unterschieden werden können, aber auch nicht im Hinblick auf die Gemeinschaft selbst. In logischer Hinsicht ist alles eins. Durch die formale Betrachtung - und logische Analysen sind immer formal - wird also der Einwand entkräftet.

\section{Kausalität bei Gremienentscheidungen-ein Scheinproblem}

Ein Nebeneffekt der Lehre von der persona moralis composita ist, dass sie den Streit über die Kausalität bei Gremienentscheidungen ${ }^{23}$ als Scheinproblem entlarvt. Das Kausalitätsproblem stellt sich vor allem für diejenigen, die für Mittäterschaft einen kausalen Beitrag jedes Mittäters verlangen. ${ }^{24}$ Auf der Grundlage der Äquivalenztheorie genügt dafür schon die (Mit-)Verursachung des gemeinsamen Tatplans, der dann seinerseits die Ursache der einzelnen Tathandlungen ist. Es ist klar, dass die Kausalität bei einer geheimen Abstimmung oder bei einer höheren Zahl von Ja-Stimmen als erforderlich Schwierigkeiten bereitet, da in beiden Konstellationen die einzelne abgegebene Stimme, zumal wenn sie unbekannt ist, nicht ohne weiteres als notwendige Bedingung für die später eingetretene Rechtsgutsverletzung angesehen werden kann. Auf der anderen Seite versteht sich dann von selbst, dass sich diejenigen, die sich der Stimme enthalten oder dagegen stimmen, nicht strafbar machen.

Für die Lehre von der persona moralis composita ist dies alles einerlei. $\mathrm{Zu}-$ nächst einmal ist die Abstimmung keineswegs das tatbestandsmäßige Verhalten, sondern der die wechselseitige Zurechnung nach $₫ 25$ Abs. 2 StGB begründende Tatplan, der von den Mittätern ja noch in die Tat umgesetzt

22 Kindhäuser, in: FS Hollerbach, 2001, S. 627 (630 f.); Jakobs, in: FS Lüderssen, 2002, S. 559 (564 ff.); van Weezel, Beteiligung bei Fahrlässigkeit, 2006, S. 163 ff.

23 Vgl. Puppe, in: Kindhäuser/Neumann/Paeffgen (Hrsg.), Nomos Kommentar, Strafgesetzbuch, 5. Aufl. 2017, Bd. 1, Vor $\$ 13$ Rn. 108, 108a m.w.N.

24 Vgl. etwa Puppe, GA 2004, 129 (131). 
werden muss. ${ }^{25}$ Allein durch eine Abstimmung ist noch gar nichts passiert. Aber die Verantwortlichkeit trifft alle an der Abstimmung Beteiligten, auch diejenigen, die sich der Stimme enthalten oder dagegen sind - weil und soweit sie sich dem mehrheitlich gebildeten Willen unterwerfen. Das Mehrheitsprinzip regelt lediglich das Zustandekommen der Entscheidung. Jeder, der dem Kollektivwillen folgt, ist Teil der persona moralis composita und damit auch als Einzelner verantwortlich. Schließlich ist es seine Wahl, zu kooperieren - statt auszutreten.

Dazu ein Beispiel: Vor einigen Jahren wurde gegen die Mitglieder einer Kammer des OLG Naumburg wegen Rechtsbeugung ermittelt. Den Ermittlungen lag der Fall „Görgülü“ zugrunde, in dem die fragliche Kammer sich mehrmals mit hanebüchenen Begründungen geweigert hatte, Urteile des EGMR oder des BVerfG umzusetzen. Schließlich traf das BVerfG die fragliche Sorgerechtsanordnung selbst und bescheinigte der Kammer, offensichtlich willkürlich entschieden zu haben. ${ }^{26}$ Damit lag der Tatbestand der Rechtsbeugung praktisch auf der Hand. ${ }^{27}$ Das OLG Naumburg verweigerte jedoch die Eröffnung der Hauptverhandlung gegen die Kollegen mit der Begründung, dass sich nur diejenigen Richter strafbar gemacht hätten, die nachweislich für die rechtsbeugende Entscheidung gestimmt hätten. Solche Feststellungen könnten aber wegen des Beratungsgeheimnisses ( $\$ 43 \mathrm{DRiG}$ ) hier nicht getroffen werden. ${ }^{28}$ In der Sache liefe diese Rechtsauffassung darauf hinaus, dass sich nur Einzelrichter nach $₫ 339$ StGB strafbar machen könnten. Die Lehre von der persona moralis composita kommt zu einem anderen Ergebnis: Auch der Richter, der die Entscheidung nicht gebilligt hat, aber die Urteilsurkunde unterzeichnet, trägt damit das Urteil mit. Es ist das Urteil des Kollegialgerichts und damit auch sein Urteil. Wenn er den rechtsbeugenden Charakter einer solchen Entscheidung kennt, macht er sich folglich auch als Mittäter strafbar. ${ }^{29}$

25 Vgl. BGHSt 37, 106 (129f.); Knauer, Die Kollegialentscheidung im Strafrecht, 2001, S. 159 ff.; Schaal, Strafrechtliche Verantwortlichkeit bei Gremienentscheidungen in Unternehmen, 2001, S. 192 ff.; abl. Sofos, Mehrfachkausalität beim Tun und Unterlassen, 1999, S. $132 \mathrm{ff} ., 156 \mathrm{ff}$.

26 BVerfG NJW 2005, 1105 ff. (einstweilige Anordnung), 2685 ff. (Hauptsacheentscheidung).

27 So auch Lamprecht, NJW 2007, 2744 (2745).

28 OLG Naumburg NJW 2008, 3585 ff.; krit. Erb, NStZ 2009, 189 ff.; Mandla, ZIS 2009, $143 \mathrm{ff}$.

29 Ebenso Sinner, in: Matt/Renzikowski (Hrsg.), Strafgesetzbuch, Kommentar, 2. Aufl. 2020, $\$ 339$ Rn. 35; Hecker, in: Schönke/Schröder, Strafgesetzbuch, Kommentar, 30. Aufl. 2019, $\mathbb{3} 39$ Rn. 3; Uebele, in: Joecks/Miebach (Hrsg.), Münchener Kommentar zum Strafgesetzbuch, 3. Aufl. 2019, Bd. 5, 339 Rn. 56. 


\section{Von der Mittäterschaft zur Beteiligung?}

Auf den ersten Blick scheint der Begriff der Zurechnung, so wie er sich in der praktischen Philosophie der Aufklärung herausgebildet hat, der Bewertung des Teilnehmers als (Mit-)Urheber der Haupttat entgegenzustehen. Causa libera ist zunächst einmal der Haupttäter allein, weil er die Tat aus freiem Willen heraus begangen hat. Da eine freiwillige Handlung per se nicht verursacht sein kann, sonst wäre sie ein notwendig stattfindendes Ereignis und eben nicht frei, ${ }^{30}$ haben weder der Anstifter noch der Gehilfe die Haupttat verursacht und sind deshalb auch keine freien Urheber dieser Tat. ${ }^{31}$

In der praktischen Philosophie der Aufklärung hat man dies jedoch anders gesehen, und wie leicht zu erkennen ist, bietet die persona moralis composita den Ansatzpunkt hierfür. Die persona moralis composita wird konstituiert durch den übereinstimmenden Willen ihrer Mitglieder, die aufgrund der Willensübereinstimmung eine Einheit bilden, der alle Handlungen zur Ausführung dieses Willens zugerechnet werden können, sowohl dem Kollektiv wie jedem einzelnen Mitglied. Dafür ist nicht erforderlich, dass jedes Mitglied der persona moralis composita selbst einen ursächlichen Beitrag geliefert hat, zumal die Vorstellung äquivalenter Kausalität im 17. und 18. Jahrhundert unbekannt war, weil man noch logisch zwischen notwendigen und hinreichenden Bedingungen unterscheiden konnte. Ein Mindestmaß wechselseitiger Abstimmung ist aber regelmäßig bei der Beihilfe notwendig - außer, wenn sie heimlich geleistet wird. Und der üblicherweise geforderte „doppelte Vorsatz" des Teilnehmers, der sich auch auf die Haupttat erstrecken muss, zeigt, dass ein mit dem Täterwillen übereinstimmender Wille vorliegt.

So argumentiert etwa der hallenser Kollege von Thomasius, Samuel Stryk in seiner Disputation „De imputatione facti alieni“ von 1688 ausdrücklich gegen eine Zurechnung fremder Schuld, die unter keinen Umständen gerechtfertigt werden könnte.

„Weil daher eine so große Verknüpfung einer Tat mit einer Person besteht, hält es unser Recht gänzlich für unbillig, des einen Schuld auf

30 S. dazu Renzikowski, in: FS Puppe, 2011, S. 201 (211 ff.). Den zugrunde liegenden Begriff der Ursache als "conditio per quam" hat Kindhäuser, in: FS Kargl, 2015, S. 253 (266 ff.); ders., in: FS Paeffgen, 2015, S. 129 ff. näher ausgearbeitet.

31 So der Kern meiner Argumentation in: Maurach/Gössel/Zipf, Strafrecht, Allgemei-

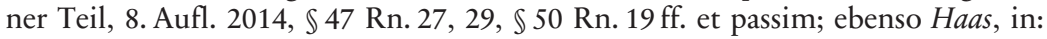
Matt/Renzikowski (Hrsg.) (Fn. 29), Vor $\$ 25$ Rn. 14, 20 ff. 
einen anderen abzuwälzen und so die Tat des einen einem anderen zuzurechnen." 32

Dabei soll es sich sogar um ein göttliches Rechtsprinzip handeln. ${ }^{33}$ Kein Problem ist dagegen der von ihm so genannte „concursus moralis“, die moralische Beihilfe, die durch Tun oder Unterlassen vor, während und auch nach der Tat geleistet werden kann. ${ }^{34}$ Der die Tat nicht selbst Ausführende kann seine Billigung ausdrücklich (voluntate expressa) oder konkludent durch seine Unterstützung (tacita) äußern. ${ }^{35}$ Als Erscheinungsformen des concursus moralis durch Tun nennt Stryk Auftrag und Befehl (mandando \& iubendo), Aufhetzung und Überredung (instigando, persuadendo), betrügerischen Rat (fraudulenter consulendo), Anerkennung (ratihabendo) und Aufnahme des Täters (receptando). ${ }^{36}$ Während diese Formen sämtlich eine ausdrückliche Willensbekundung enthalten, kann moralische Beihilfe stillschweigend etwa durch die Nutzung eines Vorteils aufgrund der Tat oder durch die Annahme einer Erbschaft begangen werden. ${ }^{37}$ Moralische Beihilfe durch Unterlassen besteht darin, eine Tat nicht zu verhindern oder nicht aufzudecken oder dem Opfer nicht die erforderliche Hilfe zu leisten. ${ }^{38}$ Es spielt keine Rolle, dass wir heute diese Handlungen anders einordnen bzw. differenzierter beurteilen. Stryk entwickelt jedenfalls eine sys-

32 Stryk, Disputatio inauguralis De imputatione facti alieni, 1688, Ingressus (A 2): "Cum itaque tanta facti cum persona connexio sit, iniqvum omnino existimarunt jura nostra, unius culpam in alterum devolvere, \& ita factum alterius alteri imputare." Näher dazu Aichele, in: Kühl (Hrsg.), Zur Kompetenz der Rechtsphilosophie in Rechtsfragen, ARSP-Beiheft 126, 2011, S. 31 (46ff.), dort auch die Übersetzung dieses Zitats.

33 Stryk, De imputatione facti alieni, I. $\$ 24$ (10): „Concordant cum hisce principiis Divina humanaque jura, qvae non admitunt ut alter ob alterius factum mereatur poenam.“ Die Vorstellung, dass Umstände nach der Tat die Zurechnung der Tat selbst begründen können, geht auf die mittelalterliche Lehre von der Teilnahme nach der Tat zurück und past natürlich nicht zum Begriff der „causa libera“; dazu näher Altenhain, Das Anschlußdelikt, 2002, S. 8 ff., 16 f.

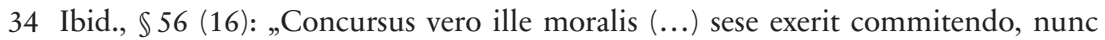
omittendo, \& utroqve modo nunc ante factum, nunc in facto, nunc post factum alterius."

35 Stryk, De imputatione facti alieni, I. $\$ 57$ (17f.): „Concurrit ergo qvis ad factum alienum I. COMMITENDO, \& qvidem vel Voluntate expressa vel tacita.“

36 Ibid., \$S 57-64 (17f.).

37 Ibid., \$\$ 65-67 (18): „Concursus vero ille moralis (...) sese exerit commitendo, nunc omittendo, \& utroqve modo nunc ante factum, nunc in facto, nunc post factum alterius."

38 Ibid., $\int \$ 68-71$ (18). 
tematische Theorie der Zurechnung, die sich auch und gerade auf zusammengesetzte moralische Personen anwenden lässt.

Stryks Begriffsbildung bleibt nicht allein. Sie findet sich in ähnlicher Weise in Baumgartens Initia philosophiae practicae von $1760,{ }^{39}$ die dann zum Vorbild von Kants Metaphysik der Sitten werden. Zwar kann der billigenden Person dieselbe Tat zugerechnet werden wie dem Täter, aber daraus folgt nicht dasselbe Maß der Bestrafung. Vielmehr ist die Zurechnung quantifizierbar, was Baumgarten weiter herausarbeitet. ${ }^{40}$ Auch das Strafgesetzbuch für das Königreich Bayern von 1813 unterscheidet zwischen verschiedenen Formen der Urheberschaft. Art. 45 lautete:

„Nicht blos I. derjenige, welcher das Verbrechen durch eigene körperliche Kraft und That unmittelbar bewirkt, sondern auch II. wer dem Vollbringer vor oder bei der Ausführung in der Absicht, damit das Verbrechen entstehe, eine solche Hülfe geleistet hat, ohne welche diesem die That nicht möglich gewesen wäre; endlich III. alle diejenigen, welche mit rechtswidriger Absicht Andere zur Begehung oder Ausführung des Verbrechens bewogen haben, sollen als die Urheber desselben bestraft werden.“ ${ }^{41}$

Eine Graduierung der Urheberschaft ist dann in den Art. 73 ff. im Detail geregelt. Man wird diesen Überlegungen nicht gerecht, wenn man sie leichter Hand als überholt abtut. Die damalige Begrifflichkeit erklärt jedenfalls viel präziser, weshalb und inwiefern ein Teilnehmer für die Haupttat mitverantwortlich ist und weshalb sich sein Strafmaß an dem der Haupttat ausrichtet, als die vorherrschende sog. ,akzessorietätsorientiere Verursachungstheorie“. ${ }^{42}$ Man könnte fast den Eindruck gewinnen, als sei die Ansicht der funktionalen Strafrechtsdogmatik damals schon vorweggenommen worden, die rechtswidrige Tat sei als „soziale Störung“ für alle Beteiligten dieselbe, ihre kollektive Leistung führe zu kollektiver Verant-

39 Vgl. Baumgarten, Anfangsgründe der praktischen Metaphysik, übers. und hrsg. v. Aichele, 2019, $\mathbb{S} \mathbb{S} 150,152$, der ebenfalls mehrere Urheber einer zusammengesetzten Tat kennt.

40 Ibid., $\mathbb{S} 152$ ff.; s. auch schon Stryk, De imputatione facti alieni, I. $\$ 72$ (19).

41 Näher dazu Eckstein, in: Koch u.a. (Hrsg.), Feuerbachs Bayerisches Strafgesetzbuch: die Geburt liberalen, modernen und rationalen Strafrechts, 2014, S. $271 \mathrm{ff}$.

42 Z.B. Joecks, in: Joecks/Miebach (Hrsg.), Münchener Kommentar zum Strafgesetzbuch, 3. Aufl. 2017, Bd. 1, Vor $\$ \mathbb{S} 26,27$ Rn. 10 ff.; Heine/Weißer, in: Schönke/ Schröder (Fn. 29), Vor $\$ \$ \$ 25$ ff. Rn. 19, jew. m.w.N.; zur Kritik Renzikowski, in: Maurach/Gössel/Zipf (Fn. 31), $\$ 50$ Rn. 13. 
wortung, ihre Rollen seien nicht qualitativ verschieden, sondern nur quantitativ graduierbar. ${ }^{43}$

Die Naturrechtslehre der Aufklärung verfügt noch nicht über eine derartig ausdifferenzierte Beteiligungsformenlehre wie wir heute mit den $\$ \$ 25$ ff. StGB. Diese Begrifflichkeiten bilden sich erst viel später heraus. Aus normtheoretischer Perspektive ist es jedenfalls ein Vorteil, das verbotene Verhalten möglichst genau zu beschreiben, und in diesem Sinne lassen sich unterschiedliche Verhaltensnormen für Täter und Beteiligte formulieren. ${ }^{44}$ Damit spezifiziert sich dann auch der Zurechnungsgegenstand.

\section{Kriminelle Vereinigungen}

Eine kriminelle Vereinigung ist gemäß $\$ 129$ StGB ein Zusammenschluss verschiedener Personen, dessen „Zwecke oder deren Tätigkeit darauf gerichtet sind, Straftaten zu begehen." Als Vereinigung gilt der auf eine gewisse Dauer angelegte organisatorische Zusammenschluss von mindestens drei Personen, die bei Unterordnung des Willens des Einzelnen unter den Willen der Gesamtheit gemeinsame Zwecke verfolgen und unter sich derart in Beziehung stehen, dass sie sich untereinander als einheitlicher Verband empfinden. ${ }^{45}$ Sieht man davon ab, dass nach der Rechtsprechung allein der gemeinsame Wille zur Begehung von Straftaten noch nicht für $\$ 129$ StGB ausreichen soll, selbst wenn einer der Beteiligten als Anführer feststeht, ${ }^{46}$ liest sich dies wie eine Definition der persona moralis composita.

Gegen diese kollektivistische Unrechtsbegründung des $\$ 129$ StGB wird gelegentlich eingewendet, dass eine Rechtsordnung rechtswidrige Organisationen nicht als Personen anerkennen könne, ${ }^{47}$ ein Vorwurf, der auch in der Diskussion über die Verbandsstrafe auftauchte. ${ }^{48}$ Doch dieser Einwand geht in die Irre. Bei der Zurechnung und dem dabei vorausgesetzten Be-

43 Vgl. Lesch, Das Problem der sukzessiven Beihilfe, 1992, S. 197 ff., 278 et passim; Jakobs, in: FS Puppe, 2011, S. 547 (555 ff.); van Weezel (Fn. 22), S. 50 ff., 60 ff.; Robles Planas, GA 2012, 276 (278 ff.); nahestehend auch Frister, in: FS Dencker, 2012, S. 119 (124 ff.).

44 S. dazu Renzikowski, in: Maurach/Gössel/Zipf (Fn. 31), \50 Rn. 19 ff.

45 Vgl. BGHSt 28, 147 (148f.); 54, 216 (221); 57, 14 (16); BGH NJW 2015, 1540.

46 S. BGH NStZ 1982, 68 f.; NJW 1992, 1518.

47 In diese Richtung etwa Lampe, ZStW 106 (1994), 683 (725); Cancio Melià, in: FS Jakobs, 2007, S. 27 (44 ff.).

48 Vgl. Jarcke, Handbuch des gemeinen deutschen Strafrechts, Erster Band, 1827, \21; Abegg, Lehrbuch der Strafrechtswissenschaft, 1836, $\$ 71 ;$ Köstlin, System des deutschen Strafrechts, $1855, \mathbb{} 401$ ). 
griff der Person geht es nicht um die Anerkennung irgendwelcher Akte als rechtmäßig bzw. rechtswirksam, sondern um das Gegenteil: Wie muss eine Entität beschaffen sein, damit man ihr bestimmte Handlungen zurechnen kann, die alsdann als rechtswidrige Taten zu würdigen sind? Die Frage nach der rechtlichen Anerkennung von Delikten ist bei der Deliktsfähigkeit von vornherein falsch gestellt.

Wenn aber gleichwohl die Mitgliedschaft in einer kriminellen Vereinigung nicht identisch mit der mittäterschaftlichen Begehung einer Straftat ist, ${ }^{49}$ wo liegt dann auf der Basis der persona moralis composita der Unterschied? Diese Frage kann man auch in umgekehrter Richtung stellen, denn die Strafbarkeit der Mitgliedschaft in einer kriminellen Vereinigung setzt nicht voraus, dass tatsächlich aus dieser Vereinigung heraus Straftaten begangen wurden. ${ }^{50}$

Was den ersten Punkt betrifft, so ist daran zu erinnern, dass die Zurechnung quantifizierbar ist. Man kann also bei Zurechnungsurteilen über die einzelnen Mitglieder einer persona moralis composita durchaus nach ihrem jeweiligen Anteil an einer bestimmten Tat gewichten. Das besondere Unrecht der Beteiligung an einer kriminellen Vereinigung besteht in der Bildung einer Unrechtsperson. $\$ 129$ StGB bestraft also die Mitwirkung an der persona moralis composita selbst. ${ }^{51}$

\section{Zur Strafbarkeit juristischer Personen}

Der bereits erwähnte Nettelbladt hat bemerkenswerterweise andernorts die Strafbarkeit juristischer Personen ausgeschlossen. So könne selbst eine gemeinsame schuldhafte Handlung aller Einzelnen, die die universitas konstituieren, der Gemeinschaft selbst nicht zugerechnet werden, da ihr weder Wille noch Verstand zukäme. Stattdessen könnten die einzelnen Mitglieder sich nur als „coauctores“, also als Mittäter strafbar machen. ${ }^{52}$ Diese Ar-

49 S. BGH NStZ 2011, 577 (578).

50 Wie etwa Köhler, Strafrecht, Allgemeiner Teil, 1997, S. 567, fordert.

51 Näher dazu Montenegro, GA 2019, 489 (497 f.).

52 Nettelbladt, Systema Elementare Iurisprudentiae Positivae Germanorum Communis Generalis, 1781, $\mathbb{S} 877$ : „(V)niversitatem ipsam numquam dolum et culpam comittere posse, cum dolus et culpa nuncquam finem communem tangant, sicque hoc respectu, deficiente intellectu et voluntate vniversitatis, et eius dolum et culpam concipi non posse: ast, quod attinet singulos ex vniversum, non solum vnus vel nonnulli ex iis, sed et omnes dolum vel culpam committere possent; licet, si omnes qui ad vniversitatem pertinent, nemine excepto, dolum vel culpam commiserunt, tale factum tamen pro facto vniversitatis cuius auctor esse debet ip- 
gumentation lässt sich freilich nicht damit vereinbaren, dass Nettelbladt an anderer Stelle einer universitas ausdrücklich Rechte und Pflichten zuschreibt, was ihre Fähigkeit zu rechtlich relevantem Tun und Unterlassen impliziert. ${ }^{53}$ Zudem widerspricht er seiner eigenen These von der logischen Ununterscheidbarkeit der einzelnen Mitglieder der universitas voneinander und von der universitas selbst (s.o.).

Die praktische Philosophie stellt also mit der persona moralis composita eine Zurechnungsfigur zur Verfügung, mit deren Hilfe jedem Mitglied des Kollektivs sowie der Kollektivperson selbst dasjenige in identischer Weise zugerechnet werden kann, was aus dem vereinigten Willen aller resultiert, der den Grund des Zurechnungsurteils darstellt. ${ }^{54}$ Eine Zurechnung der entsprechenden einzelnen, gemäß des einheitlichen Willens vorgenommene Handlung an die derart bestimmte persona moralis composita - und damit auch ihre Bestrafung - ist damit prinzipiell möglich.

Bei großen Unternehmen wie Aktiengesellschaften, multinationalen Konzernen usw. kann man sich freilich fragen, was der einzelne Aktionär oder auch der einzelne Monteur am Fließband mit den Sünden des Vorstands zu tun haben. Obwohl diese Sünden nicht aus einer Willensübereinkunft mit diesen Leuten hervorgegangen sind, obwohl sie nicht selbst gehandelt haben und also für die Begehung der Straftat nichts können, werden sie durch eine Milliardengeldbuße ebenfalls getroffen, auch wenn

sa vniversitas, habere necquit, sed pro facto tali singulorum ex vinversitate, cuius coauctores sunt omnes que universitatem constituunt.“ - „Die Gemeinschaft selbst kann niemals vorsätzlich oder fahrlässig verbrechen, weil Vorsatz und Fahrlässigkeit nie das gemeinsame Ziel betreffen, und damit in dieser Hinsicht durch einen Mangel an Einsicht und Wille der Gemeinschaft es ihr nicht möglich ist, Vorsatz und Fahrlässigkeit überhaupt zu fassen. Indes soweit es die Einzelnen aus der Gemeinschaft betrifft, können sie nicht nur allein oder als wenige, sondern auch alle vorsätzlich oder fahrlässig verbrechen. Es ist möglich, dass wenn ausnahmslos alle, die zur Gemeinschaft gehören, vorsätzlich oder fahrlässig verbrechen, eine solche Tat dennoch für eine Tat der Gemeinschaft angesehen wird, deren Urheber selbst der Gemeinschaft verbunden ist. Sie kann nicht dafür gehalten werden, sondern für die Tat solcher Einzelner der Gemeinschaft, die alle als Mittäter die Gemeinschaft begründen.“ S. auch Haas, in: Kaufmann/Renzikowski (Hrsg.) (Fn. 20), S. 133.

53 Nettelbladt, Systema elementare universae jurisprudentiae naturalis (Fn.52), $\$ \$ 83 \mathrm{f}$. und $373 \mathrm{ff}$.

54 M.a.W.: Die persona moralis composita eignet sich als Grundlegung einer Theorie über „Systemunrecht und Unrechtssysteme“ (Lampe, ZStW 106 [1994], 683 ff.). Die beliebte Behauptung, der Freiheitsbegriff der Aufklärung habe die Straf- und Schuldfähigkeit von Verbänden ausgeschlossen, so Dust (Fn. 12), S. 40 f. m.w.N. ist daher falsch. 
sie formal-rechtlich nicht die Adressaten der Sanktion sind, sondern das Unternehmen. Von einer Bestrafung für fremde Schuld kann jedoch keine Rede sein, denn die Sanktion greift nicht in ihre Rechte ein. Anteilseigner und Arbeitnehmer sind rechtlich nicht anders betroffen als etwa die Mitglieder einer Familie, deren wirtschaftliche Grundlage möglicherweise durch die Inhaftierung eines Familienmitglieds entzogen wird. Man könnte sogar erwägen, ob sie nicht insofern sogar Mitglieder der persona moralis composita sind, indem sie ihr Vermögen oder ihre Arbeitskraft investiert und es dem Unternehmen dadurch erst ermöglicht haben, das fragliche Delikt zu begehen. Denn die Zurechnung ist graduierbar, gewissermaßen von 100 für den Täter bis zu 0,1 für den entferntesten Mitläufer. Die imputatio iuris bezieht sich zudem nicht ausschließlich auf Strafe im klassischen Sinn, sondern als Sanktion kann auch eine zivilrechtliche Vermögenseinbuße aufgefasst werden. Und die Zurechnung in der leichtesten Form erklärt, dass keine völlig Unbeteiligten getroffen werden.

\section{Einwände}

Einige Bedenken gegen die Begründung der Verbandssanktion mit der Zurechnungsfigur der persona moralis composita habe ich bereits an anderer Stelle angesprochen. ${ }^{55}$ Auf sie möchte ich hier nicht noch einmal eingehen. Stattdessen sollen zwei weitere Probleme erörtert werden.

\section{a) Das Problem der Identität}

Unternehmen verändern sich: Bestandteile werden veräußert, neue Firmen hinzugekauft, nach dem Rücktritt des Aufsichtsratsvorsitzenden wird ein neuer gewählt, die Aktionäre ändern sich ständig usw. Damit verbunden ist das Problem, dass etwa die Anteilseigner, die zum Zeitpunkt der fraglichen Straftat am Unternehmen beteiligt waren, nicht mehr von einer Jahre später verhängten Sanktion betroffen sind, weil sie ihre Anteile inzwischen mit Gewinn veräußern konnten, bevor der Kurs des Unternehmens eingebrochen ist.

Aus diesem Grund wendet man gegen ein Verbandsstrafrecht ein, dass einem Unternehmen diese Identität fehlt. Gewissermaßen wird ein ande-

55 Renzikowski, GA 2019, 149 (153 f.: Juristische Person als Fiktion; 156 ff.: Höchstpersönlichkeit der Strafe). 
rer bestraft als derjenige, der die Straftat begangen hat. ${ }^{56}$ In der Tat wäre das nichts anderes als eine illegitime Bestrafung für fremde Schuld. Dieses Problem lässt sich dann lösen, wenn man zwischen den essentiellen Merkmalen und den Akzidentien unterscheidet. Für eine juristische Person ist es essentiell, dass sie Organe hat wie einen Vorstand, oder auch ein Vermögen. Es ist aber nicht essentiell, wie die Organe personell zusammengesetzt sind. Ein Verein wird nicht dadurch „ein anderer“, dass der Vorsitzende durch eine Wahl ausgewechselt wird. Die VW-AG wird nicht dadurch „eine andere“, dass sie künftig auch Elektroautos produziert. Grundsätzlich bestimmt sich die Identität einer juristischen Person nach Gesellschaftsrecht. ${ }^{57}$

Im Übrigen ist es, wie bereits ausgeführt, ja auch nicht der physische Körper, der eine Person ausmacht, sondern die freie Willkür, genauer: das Vermögen, die eigenen Handlungen nach Maximen zu bestimmen. Irgendetwas Physisches ist nur deshalb erforderlich, weil ein Wille allein keine Veränderung in der Außenwelt bewirken kann, aber nichts spricht dafür, dass dieser Körper stets dieselbe unveränderte Beschaffenheit haben muss. Dieser Einwand trägt also nicht.

\section{b) Unzulässige Doppelbestrafung}

Der Vorwurf einer unzulässigen Doppelbestrafung (vgl. Art. 103 Abs. 3 GG) lässt sich in zwei Richtungen erheben: Entweder erledigt die Bestrafung der einzelnen Mitglieder der persona moralis composita die parallele Sanktionierung des Verbands als solchen oder die Strafe gegen den Verband erledigt parallele Sanktionen gegen seine Mitglieder. ${ }^{58}$ Die logische Ununterscheidbarkeit der einzelnen Mitglieder der persona moralis composita voneinander und von der Gesamtheit steht der vordergründigen Ausflucht entgegen, zwischen dem Individuum und dem Kollektiv als verschiedenen Subjekten zu unterscheiden.

Dennoch kann dieser Einwand entkräftet werden. Strafe ist dem Begriff nach ein Übel, welches den Täter für seine unrechte Tat trifft und, darin besteht der Grundgedanke des Talionsprinzips, dem „Wesen“ seiner Tat

56 S. etwa Sola, GA 2016, $625 \mathrm{ff}$.

$57 \mathrm{Zu}$ den damit verbundenen Problemen s. Rogall, in: Mitsch (Hrsg.), Karlsruher

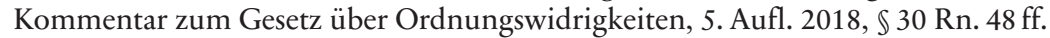

58 In diese Richtung Jakobs, in: FS Lüderssen (Fn. 22), S. 564 ff.; Kindler, Das Unternehmen als haftender Täter, 2008, S. $282 \mathrm{ff}$. 
entspricht. ${ }^{59}$ Das Wesen des Verbrechens besteht nun darin, dass der Straftäter seine Freiheit missbraucht und dadurch die Gesetze der Freiheit verletzt. Auf diese Weise schließt sich der Straftäter in gewisser Weise von der Teilhabe an der bürgerlichen Gesellschaft aus. ${ }^{60}$ Mit der Strafe wird dem Straftäter dasselbe zurückgegeben: Strafen sind eine Weise, den Straftäter von der vollen Teilhabe an der Gesellschaft durch eine Einbuße seiner Rechte auszuschließen.

Die Strafe muss aber nicht nur dem „Wert" der Straftat, sondern auch der Eigenart des Zurechnungssubjekts entsprechen. Das lässt sich an einem Vergleich der Bestrafung von Mittätern und Verbänden exemplifizieren. Die gemeinsam begangene Tat ( $\$ 25$ Abs. 2 StGB) wird dem Kollektiv und damit jedem einzelnen Komplizen in gleicher Weise zugerechnet (s. oben III.1.). Die Bestrafung erschöpft sich jedoch in der Sanktionierung jedes Mittäters, denn darüber hinaus, d.h. ihre physischen Körper (Freiheitsstrafe) oder ihr Vermögen (Geldstrafe) ist schlichtweg nichts mehr übrig, was noch von einer Sanktion erfasst werden könnte. Bei einem Unternehmen ist das jedoch anders. Neben den Verantwortlichen, die in eigener Person zur Rechenschaft gezogen werden, stehen die ökonomischen Ressourcen des Unternehmens zur Verfügung, die ja erst die Straftat ermöglicht haben. Die ökonomische Sanktion gegen das Unternehmen entspricht dem Wert dieser Tat und ist einerseits ein Eingriff in die ihm eingeräumte wirtschaftliche Betätigungsfreiheit (vgl. Art. 12 GG) und entzieht ihm andererseits wirtschaftlichen Nutzen aus dieser Straftat. Eine doppelte Bestrafung liegt hierin ebenso wenig wie in der Möglichkeit, gegen einen Einzelnen neben der Hauptstrafe (z.B. Geldstrafe) eine Nebenstrafe (z.B. Fahrverbot) zu verhängen.

59 Nach Hegel das "Wesentliche, was der Verbrecher verdient hat" (Grundlinien der Philosophie des Rechts, 1821, $\$ 101)$. Ähnlich heißt es bei Kant, MdS, AA VI, S. 363: „Nur dann kann der Verbrecher nicht klagen, daß ihm unrecht geschehe, wenn er seine Übeltat sich selbst über den Hals zieht und ihm, wenngleich nicht dem Buchstaben, doch dem Geiste des Strafgesetzes gemäß das widerfährt, was er an anderen verbrochen hat."

60 Nach Kant, MdS, AA VI, S. 331 macht eine Tat, die sich gegen das ,gemeine Wesen“, d.h. die rechtlich verfasste Gesellschaft selbst richtet, denjenigen, der sie begeht, „unfähig ..., Staatsbürger zu sein“. S. dazu auch Ripstein, JRE 16 (2008), 238 (250 ff.). 


\section{Schluss}

Die in der Aufklärung entwickelte Figur der persona moralis composita zeigt, inwieweit Kollektive als Zurechnungssubjekte angesehen werden können. Aus dieser Analyse folgt allerdings keineswegs, dass der Gesetzgeber diese Begrifflichkeit aufgreifen und in das Strafrecht inkorporieren muss. Einerseits ist die Beteiligungsformenlehre unserer heutigen Strafrechtsdogmatik auf eine etwas andere Weise ausdifferenzierter als die Vorstellungen des 18. Jahrhunderts. Zudem finden sich rechtsvergleichend noch ganz andere Modelle der Verteilung der Verantwortlichkeit auf verschiedene Beteiligte. Andererseits sind alle möglichen Gründe denkbar, auf ein eigenes Unternehmensstrafrecht zu verzichten. Ebenso wenig ist vorbestimmt, wie ein solches Verbandsstrafrecht konkret ausgestaltet sein muss. Lediglich seine systematisch stimmige Möglichkeit sollte hier aufgezeigt werden. 